\title{
Attention and nontarget effects in the location-cuing paradigm
}

\author{
GARVIN CHASTAIN \\ Boise State University, Boise, Idaho \\ MARYLOU CHEAL \\ University of Dayton Research Institute, Higley, Arizona \\ and Arizona State University, Tempe, Arizona \\ and \\ DON R. LYON \\ University of Dayton Research Institute, Higley, Arizona
}

\begin{abstract}
Three experiments were conducted in order to determine whether irrelevant items presented outside the focus of attention would affect the identification of a precued target. A peripheral cue indicated one of eight possible locations in a circular array, centered on fixation with a radius of $5.25^{\circ}$. After a variable interval (0-200 msec), eight characters were presented briefly and masked. In each experiment, there was an effect of the identity of the characters at the seven noncued locations (the nontargets) on the accuracy of identification of the target. When there were more nontargets identical to the target, accuracy was higher than when there were fewer nontargets identical to the target. Nontargets consistently affected performance despite incentives to focus only on the target.
\end{abstract}

Can an irrelevant item presented outside the focus of attention affect performance on an attended target? The literature is inconclusive as to when, how, and even whether such an effect occurs. Johnston and Dark (1986), in a brief review, concluded that processing outside of an area of focused attention is restricted primarily to simple physical features - the kind that might be perceived by automatic processes. Eriksen, Webb, and Fournier (1990) have suggested that confusion in interpretation of experiments meant to settle this question may be due to the confounding of two separate, nonsequential attention processes: an automatic process and a focused process. Their idea is that when an observer focuses attention on a target, the focal process can proceed independently of any automatic processing that is occurring simultaneously or that has occurred recently. Automatic processing may occur, but the results of that process may be overridden by the focal process.

Automatic processing is not the only reason why irrelevant items could affect performance. These items may in fact be attended (selected) and then identified (Yantis \& Johnston, 1990), particularly when the items are potential

The authors thank A. Kramer for helpful suggestions and comments on an earlier version of the manuscript. Consultation on statistical analyses was received from $D$. Hubbard. The research was partially funded by the Air Force Office of Scientific Research (Life Sciences Task 2313T3) and the Air Force Armstrong Laboratory (Contract F-33615-90-C-0005). Support was also provided by the Department of Industrial and Systems Management Engineering and the Department of Psychology at Arizona State. Correspondence concerning this article should be addressed to M. Cheal, 127 E. Loma Vista Drive, Tempe, AZ 85282-3574 (e-mail: cheal@imap1.asu.edu). targets. Miller (1987) presented three letters in each array, with the middle letter always to be reported and the outer letters ignored; outer letters were always neutral; that is, they were never possible targets. Performance was influenced by the frequency with which particular outer letters appeared with particular targets, which suggested that some perceptual processing of the outer letters occurred and that these letters were associated with certain responses. However, Paquet and Lortie (1990) found that uncertainty regarding precise target location was partly responsible for the effect, which suggested that there was some difficulty in maintaining attention only on the target. When target location was precued, there was less effect of congruity between the target and the outer letters. The fact that congruity effects were not completely eliminated was attributed to imperfect focusing of attention, which would not be surprising, inasmuch as there was less than $1^{\circ}$ of separation between the letters (Eriksen \& St. James, 1986).

Yantis and Johnston's (1990) precuing procedure included much stronger controls to prevent the misfocusing of attention. They used a circular array of eight letters, one of which was precued for either 100 or $200 \mathrm{msec}$ before the array appeared. Edge-to-edge intercharacter distance was over $2^{\circ}$ (to allow attention to be focused on only one letter; see Eriksen \& St. James, 1986). Both the location and the identity of the target changed from trial to trial. On redundant-target trials, one of the seven uncued letters was identical to the cued letter, with the other six letters unique. On nonredundant-target trials, none of the uncued letters were identical to the cued letters, although the seven uncued letters were unique. No difference in performance was found between redundant- and nonredundant-target trials. 
Pashler and Badgio (1985), on the basis of their own results, suggested that it was possible that in displays of this type, "parallel encoding processes are capable of extracting the identities present in a multielement display but not of tying those identities to locations" (p. 119). Therefore, identities are derived regardless of where or whether attention is directed, but an item thus identified is available for report only as attention is directed to its location. Perhaps evidence for the presence of a particular identity accumulates with the number of items having this identity, and therefore, the determination of this identity for a cued item is facilitated. However, others have not found evidence of recognition of unattended items (Wright, Katz, \& Hughes, 1993). Also, Yantis and Johnston (1990) found no improvement in performance on redundant-target trials, and no effect of the distance between the identical letters, suggesting that if attention is successfully focused, the redundant uncued letter does not enhance performance even if it is adjacent to the cued letter.

Even with the careful work of Yantis and Johnston (1990), some questions are left unanswered. Specifically, the difference between having 0 of 7 and 1 of 7 nontargets identical to the target (MATCH-T) might not be great enough to influence performance significantly when a single target location is precued. In addition, only two precuetarget delays, 100 and $200 \mathrm{msec}$, were used, although accuracy of responding to a precued target improves dramatically between 0 and $100 \mathrm{msec}$ (Cheal \& Lyon, 1989). It would be interesting to introduce shorter delays, beginning at $0 \mathrm{msec}$, in search of an interaction between delay (interstimulus interval, or ISI) and MATCH-T.

Furthermore, the results of these experiments may help us to differentiate between different concepts of the manner in which attention is allocated to the visual field. For instance, if attention is allocated according to a spotlight metaphor (Posner, 1980), so that attention starts at fixation and moves directly to the cued location, then nontargets should have no effect on responses. However, if attention allocation varies in area as does a zoom lens (Eriksen \& St. James, 1986), at very short ISIs when attention is widely distributed across the display, there may be an effect of MATCH-T, which decreases and eventually disappears as ISIs become longer and attention is narrowly focused on the target location.

On the other hand, this change with ISI may not occur if one conceptualizes attention as allocated in a gradient (LaBerge \& Brown, 1986, 1989) that changes over time (gradient-filter metaphor for attention; Cheal, Lyon, \& Gottlob, 1994). In the gradient-filter metaphor, attention is conceived as modulating the permeability of multiple filters over the visual field. These filters regulate the flow of information. With increasing attention at a filter, that filter becomes more permeable and the flow of information increases. The permeability of individual filters can be independent of others unless the total capacity of attentional resources has been used. In that case, for greater permeability in one area, there must be a decrease in another region. Thus, this is a flexible system that does not necessarily predict either an interaction of MATCH-T with ISI or a lack of such interaction.

In preliminary experiments in our laboratory, displays with four possible target locations were used. Data from these experiments provided weak evidence for a MATCH$T$ effect. To strengthen the evidence, in this article we report three experiments, each involving circular displays of eight items, ISIs of 0-200 msec, and MATCH-T somewhere between zero and seven items within each display. Accuracy, rather than response time, was the dependent measure, and the stimuli were plus signs, each with one arm removed (these characters are the same as a capital $\mathrm{T}$ in rotated orientations; they are not called Ts in this paper to avoid confusion with the term MATCH-T, which refers to a nontarget matching a target).

In Experiment 1, subjects were required to report target identity (the direction of the arm opposite the one removed on the target) and the location of the target. Accuracy increased both with increases in MATCH-T and over ISIs, with no significant interaction between these variables. The pattern of results was the same with or without the inclusion of incorrect location-response trials. In Experiment 2 , the number of nontargets different from the target but identical to each other (MATCH-NT), as well as MATCH-T, was varied. The idea was that the increases in MATCH-NT would increase the strength of a competing response. Accuracy again increased with increases in either ISI or MATCH-T, and as in the first experiment, there was no significant interaction between ISI and MATCHT. However, within a given MATCH-T, there was no effect of MATCH-NT on accuracy, although there was a significant effect on which a particular response was produced in error.

One possible explanation for these results is that observers were occasionally attending to nontarget locations. In Experiment 3, only one nontarget matched the target on $87.5 \%$ of the trials. This should have produced an incentive to ignore nontargets, because on most trials the identity of six of seven nontargets was different from that of the target. Despite this incentive, accuracy was much higher with MATCH-T $=6$ than with MATCH-T $=1$.

\section{EXPERIMENT 1}

Preliminary data suggested that when three nontargets in a display matched the target, responses were more likely to be accurate than when fewer characters matched the target. Furthermore, when seven nontargets matched the target, accuracy was $22 \%$ higher than when one nontarget matched the target (a MATCH-T effect; mean proportion correct for 12 subjects: .739 and .578 for 7 and 1 matching nontargets, respectively). One possible difficulty in interpretation of these data is that observers may not have used the cue on all trials. It is possible that a MATCH-T effect resulted because, on some trials, observers oriented to an uncued location and responded to a nontarget. In order to reduce the possibility that subjects were orienting to an incorrect location on some trials, in 
Experiment 1, they were required to indicate the location of the cue in addition to giving the response as to the identity of the target.

Thus, Experiment 1 was conducted (1) to test the robustness of a MATCH-T effect with a display of eight characters when the number of nontargets that matched the target (MATCH-T) was varied from zero to seven, and (2) to require subjects to indicate the target location after giving an identification response. Data from trials in which some location responses were incorrect were compared with data from which incorrect location responses were eliminated prior to statistical analysis.

\section{Method}

Subjects. Different subjects for each of these experiments were drawn from the introductory psychology subject pool at Boise State University and received extra credit for their participation. Most were 1st-year students (mean age, 20.1 years). In this experiment, the 16 subjects ( 5 men and 11 women) reported normal or correctedto-normal visual acuity.

Apparatus. Stimuli were presented on a Zenith ZCM-1490-Z analog display controlled by an IBM PS/2 Model 30 . The decay rate of the P-22 phosphor on the display was to $10 \%$ within $1 \mathrm{msec}$. Distance of the subjects' eyes from the display was maintained at approximately $38 \mathrm{~cm}$ by a Gulf \& Western table-mounted chinrest with head restraint. Eye movement was monitored continuously by the experimenter with a tripod-mounted box camera connected to a television set.

Stimuli. The target display contained eight modified plus signs, with the critical arm (arm opposite the one removed) pointing up, down, to the left, or to the right. Complete plus signs from which the targets were taken subtended a visual angle of approximately $0.8^{\circ}$ vertically and horizontally. Identification of this type of character requires serial search in a standard visual search task (Cheal \& Lyon, 1992). The eight modified pluses shown on each trial appeared more than $3^{\circ}$ of visual angle apart and were equally spaced around the circumference of an imaginary circle, centered on fixation with a radius of $5.25^{\circ}$; target locations were at $0^{\circ}, 45^{\circ}, 90^{\circ}, 135^{\circ}, 180^{\circ}, 225^{\circ}$, $270^{\circ}$, and $315^{\circ}\left(120^{\prime}\right.$ clock $\left.=0^{\circ}\right)$. One location was precued with a $0.45^{\circ}$ square, appearing approximately $4.25^{\circ}$ from fixation. Masks, present at each of the eight locations, followed the outline of a complete plus sign.

Experimental Design. The target, which always appeared at the cued location, was presented at each of the eight locations equally often, with the critical arm pointing in each direction on an equal number of trials at each location. The interstimulus interval (ISI) was $0,50,100$, or $200 \mathrm{msec}$. On each trial, $0,1,4$, or 7 nontargets were identical to the targets (MATCH-T). These variables and levels were combined factorially ( 8 locations $\times 4$ ISIs $\times 4$ MATCH-Ts $\times 4$ target orientations) to produce 512 trials per subject, with combinations presented in a pseudorandom order during each session.

Procedure. Each subject served in a single session of approximately $1 \mathrm{~h}$. The subject gazed at the fixation dot which appeared for $500 \mathrm{msec}$ in the center of the screen at the beginning of each trial. The precue then appeared with enhanced brightness for $16.7 \mathrm{msec}$. After the ISI, a potential target character appeared at each of the eight possible locations for $50 \mathrm{msec}$. The masks followed immediately and remained on the screen until the subject pressed the up, down, left, or right arrow key to indicate the direction of the arm opposite the one removed on the target. The target was the character at the cued location. Subjects responded as to the identity of the target on the numeric keyboard and then gave a location response after each identification response at the end of each trial. Subjects made the location response by pressing the number keys on the numeric keypad to indicate one of the eight locations in the display (with the " 8 " key indicating the top location on the screen, and the remaining keys corresponding to the locations of items around the circumference of the imaginary circle that passed through them).

A "happy face" appeared if the response was correct, or a minus sign if it was incorrect, for $1 \mathrm{sec}$. The next trial began after an intertrial interval of approximately 2 sec. The subject could pause at any time merely by withholding the response until ready. To ensure that the subject kept his/her eyes at the correct distance from the screen and fixated its center, a chinrest with head restraint was used and the eyes were monitored. If an eye movement occurred immediately before, during, or immediately after the trial, the subject was admonished by the experimenter.

Eye movements could have had little influence on the results because (1) eye movements occurred on less than $1 \%$ of the trials, and (2) the precue plus target exposure duration was such that eye movements to the target were possible only at the longest stimulus onset asynchrony.

\section{Results and Discussion}

This experiment replicated the results of our earlier research in conditions in which subjects correctly localized the target. Responses were more accurate when more targets matched the target than when fewer matched. There was an average of 34 errors in locating the target out of 512 trials per subject $(6.6 \%$ of all trials). A first analysis of variance (ANOVA) was conducted on all data, including data from trials on which the location response was incorrect. For a second ANOVA, the mean proportion of responses correct at each ISI and MATCH-T for each subject were used only for trials on which the location response was correct (Figure 1). The effects that were significant were the same as in the first analysis: ISI $[F(3,45)=9.07$, $\left.M S_{\mathrm{e}}=.0117, p<.001\right]$ and MATCH-T $[F(3,45)=13.27$, $\left.M S_{\mathrm{e}}=.0232, p<.001\right]$; there was no significant interaction $[F(9,135)=0.97, p>.10]$.

Tukey (HSD) analysis of data without location errors indicated the following significant differences $(p<.05)$ : MATCH-T $=0(M=0.539)$ was less than MATCH-T $=4$ $(M=0.633)$ or MATCH-T $=7(M=0.704)$; and MATCH-T $=1(M=0.594)$ was less than MATCH-T $=7$. Tukey (HSD) analysis of data that included trials on which location errors were committed yielded an identical pattern of

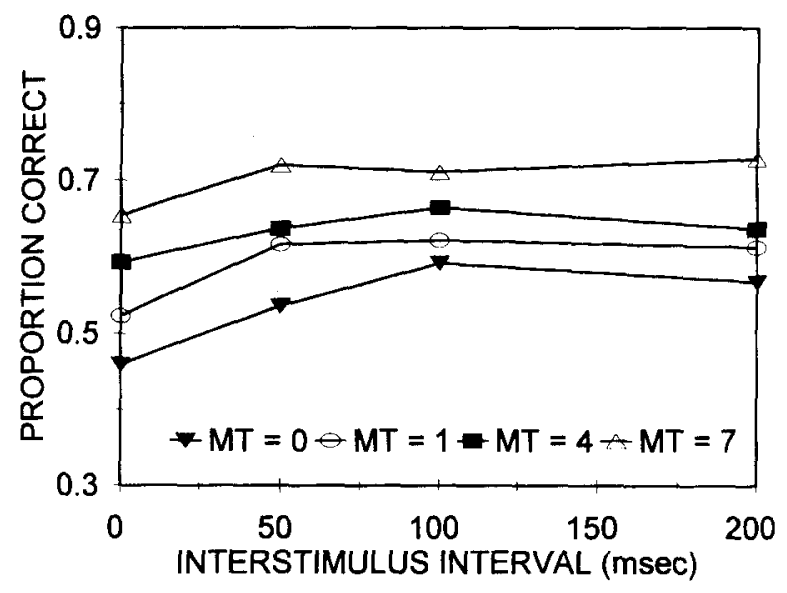

Figure 1. Mean proportion correct for each MATCH-T and interstimulus interval of only trials in which location responses were also correct in Experiment 1. 
significant effects. There was no effect of the number of location errors across MATCH-T $[F(3,45)=1.35, p>.10]$.

Although only $6.6 \%$ of responses involved a location error, a comparison was made through a general linear models analysis of MATCH-T and ISI effects on trials with versus trials without location errors. Although accuracy was much better on trials without a location error $(M=$ 0.618 ) than on only those trials in which a location error was made $(M=0.339 ; p<.001)$, no significant interaction that included the location error variable was found.

The significant increase in accuracy with increasing ISI suggests that attention was being focused at the target location. Also, with MATCH-T $=1$, there was no significant distance effect in location errors when the MATCH-T was 1 versus 2 versus 3 versus 4 characters from the target $[F(3,45)=0.37, p>.10]$, nor with MATCH-T $=4$ when the closest MATCH-T was one character away versus two characters away $[t(15)=0.36]$.

A further analysis of data from trials with only correct location responses was made to determine whether the significant effect of MATCH-T was due to correct location responses that were made by chance. Because eight locations are in the display, on one eighth of the trials on which the subject is completely unsure of the location, the location response could be correct by chance.

If the target location were judged incorrectly, the subject would attend to a location different from the target location. The proportion of identification responses that were correct could be adjusted to reflect trials on which the incorrect location was attended, but afterwards the location was guessed correctly for the location response. With MATCH-T $=0$, the character in any nontarget location could not match the target, and therefore the identification response was always incorrect if the subject attended a nontarget location. Therefore, for MATCH-T $=0$, accuracy was adjusted upward. Since one eighth of all apparently correct location responses should, on the average, represent a guess, one eighth of the incorrect identification responses were changed to correct when the location response was correct. With MATCH-T $=7$, the nontarget characters always matched the target, and therefore the identification response was always correct when the subject attended a nontarget location. Therefore, for MATCH-T $=7$, the proportion of identification responses was adjusted downward appropriately by changing to incorrect one eighth of the correct identification responses when the location response was correct (but probably only guessed correctly). The difference between the adjusted proportions for MATCH-T $=0$ and MATCH-T $=7$ remained significant $[t(15)=3.18$, $p<.01]$. This is a very conservative test, but it is still possible that on some trials the subject knew the correct location and reported it in the location response, but nevertheless attended to a character at a different location.

Because of the possibility that the MATCH-T effect was due to subjects attending to a location other than the one containing the target on some trials, the number of nontargets that differed from the target but were identical to each other (MATCH-NT) was controlled in Experiment 2. This was in addition to controlling MATCH-T.

\section{EXPERIMENT 2}

If evidence for a particular response accrues as the number of identical characters in the display increases, incorrect responses should increase with the number of nontargets different from the target but identical to each other (MATCH-NT). Suppose that, with eight items in the display, MATCH-T $=1$. This means that six items are different from the target. If all six of these items are identical, the evidence for that response should be greater than the evidence when two items share each of the three identities that are different from that of the target, or even when three items share each of two identities that are different from that of the target. However, if on some trials a single item other than the target is selected, MATCH-T but not MATCH-NT should be reflected in target identification accuracy.

\section{Method}

Thirty subjects (14 men and 16 women), none of whom had served previously, participated in this experiment. The design was the same as in Experiment 1, with two exceptions: (1) In order to maintain a factorial design and keep the experiment within the time limits available ( $50 \mathrm{~min}$ per subject), cues and targets were presented only at $0^{\circ}, 90^{\circ}, 180^{\circ}$, or $270^{\circ}$, although there were always characters at the eight locations. (2) The number of nontargets different from the target but identical to each other was carefully controlled. As in the first two experiments, MATCH-T was $0,1,4$, or 7 . However, when MATCH-T $=0$, MATCH-NT was 7 , or 2,2 , and 3 (for each of the three possible responses other than that indicated by the target). When MATCH-T $=1$, MATCH-NT was 6 , or 3 and 3 , or 2, 2, and 2. When MATCH-T = 4, MATCH-NT was 3, or 1,1 , and 1 . Finally, when MATCH-T $=7$, every item in the display was identical. ISI again was $0,50,100$, or $200 \mathrm{msec}$, and this variable was factorially combined with each MATCH-NT within each MATCH-T, producing 16 trials for each combination or 512 trials in all. The order of trials representing the combination of variables was completely randomized for each subject. Location responses were not made.

\section{Results and Discussion}

MATCH-T effects. As in the first experiment, eye movements occurred on less than $1 \%$ of all trials. The first analysis for this experiment was of MATCH-T; MATCHNT was not considered. There was a significant increase in accuracy with increasing ISI $\left[F(3,87)=42.21, M S_{\mathrm{e}}=\right.$ $.0138, p<.001]$ and with increasing number of MATCHTs $\left[F(3,87)=19.76, M S_{\mathrm{e}}=.0081, p<.001\right]$. (See Figure 2.) The fact that the MATCH-T effect was apparently smaller than in the previous experiments may have been due to fewer possible target locations in this experiment (only four) than in Experiment 1 (eight possible target locations). The interaction between the two variables was not significant $[F(9,261)=1.20, p>.10]$.

Tukey (HSD) analysis of the data indicated that all MATCH-T differences were significant $(p<.05)$ except for MATCH-T $=0$ versus MATCH-T $=1$ and MATCH-T $=1$ versus MATCH-T $=4$. Means are presented in column 2 of Table 1. The significant increase in accuracy with increases in ISI suggests that attention was being focused at the target location. There was no significant effect of the distance of MATCH-T from the target for 


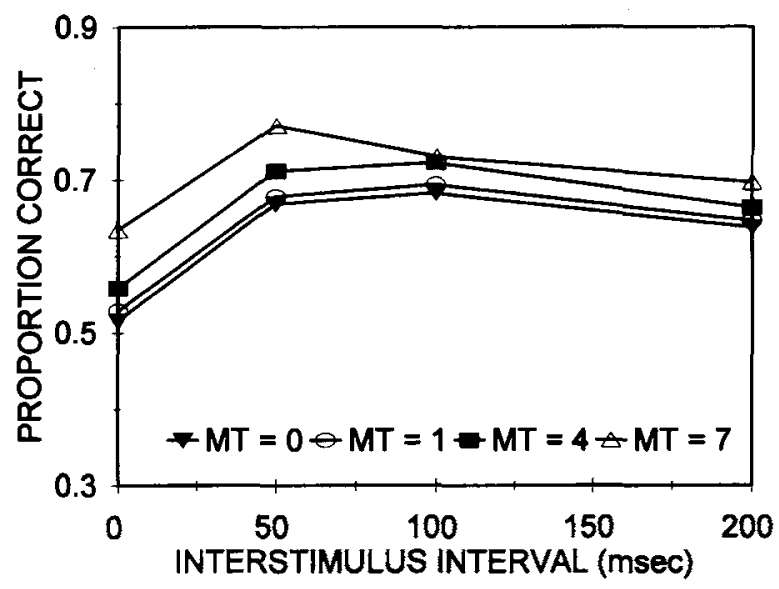

Figure 2. Mean proportion correct for each MATCH-T and interstimulus interval in Experiment 2.

MATCH-T $=1[F(3,87)=0.62, p>.10]$, or for MATCH-T $=$ $4[t(29)=0.47]$.

MATCH-NT effects. A complete analysis was conducted with MATCH-NT within MATCH-T separated to form a total of eight levels of MATCH-NT (means of proportion correct for MATCH-NT are reported in column 4 of Table 1). The overall comparison was significant $\left[F(7,203)=7.16, M S_{\mathrm{e}}=.0133, p<.001\right]$. There was, however, no effect of MATCH-NT within any one MATCH-T. Tukey (HSD) analysis showed that none of the MATCH-NT means within a given MATCH-T differed significantly.

Even though there was no effect of MATCH-NT that was independent of MATCH-T, it is possible that MATCHNT had an effect on incorrect responses. Given that the observer did not give the correct target response, would the most likely response (in error) be to the character that was represented most frequently in the display? To answer this question, the data were analyzed trial by trial. For each observer, the proportion of trials in which the response corresponded to the identity of each MATCH-NT for each MATCH-T and to each response that was to a character not in the display (called MATCH-NT $=0$ ) was computed (responses that were, of course, in error). These proportions, given in Table 1, column 6 , as $p$ (responses that match each MATCH-NT), increased (see column 3, Table 2) with an increasing number of matching nontargets (MATCH-NT, Table 2, column 2). This MATCH-NT effect was independent of MATCH-T. The items within any one MATCH-NT did not differ significantly [MATCH$\mathrm{NT}=0, F(4,116)=0.17 ;$ MATCH-NT $=2, F(1,29)=$ 0.15 ; $\mathrm{MATCH}-\mathrm{NT}=3, F(2,50)=1.32$; all n.s.].

Next, the proportion of error responses [ $1-p$ (corr)] was partitioned among MATCH-NT within each MATCH-T [ $p$ (matching error responses), column 7 of Table 1]. Note that when MATCH-NT is listed in increasing order in Table 2, not only are the proportions of the responses that match each MATCH-NT in increasing order (column 3), but also the proportion of the error responses that match each MATCH-NT (column 4) are generally in increasing order. Thus, although the MATCH-NT did not have an effect on accuracy independent of MATCH-T, there was a significant proportion of trials in which, given an error, the observer responded with the nontarget that was represented most frequently in the display that was presented [ANOVA across the 13 proportions in column 4 of Table 2 , $\left.F(12,348)=15.11, M S_{\mathrm{e}}=.0015, p<.0001\right]$.

Finally, the partitioning of error responses among MATCH-NT led to the following descriptive equation:

$$
p(\text { corr })=1-[.3+.01(\text { MATCH-NT })] .
$$

The .3 inside the parentheses represents an empirically derived baseline guess rate of .1 for each of the 3 responses that are not the target response (see Table 2, column 3, lines 1-5). The .01 per MATCH-NT is a value also derived from column 3 of Table 2. First, the means for $p$ (responses that match each MATCH-NT) for each MATCH-NT were computed. Then the difference was taken between the mean for each increase in MATCH-NT. The mean of these differences equals .01. Values derived from Equation 1 appear in column 5 of Table 1 . Note that they are very similar to the actual data.

\section{EXPERIMENT 3}

It is possible, in the preceding experiments, that subjects intentionally processed the nontargets, at least on a proportion of the trials. When many of the nontargets match the targets, this strategy might produce inflated accuracy. This experiment, therefore, was run to ensure that subjects were unlikely to direct their attention intentionally to the nontargets.

In Experiment 1, 50\% of the trials involved multiple nontargets that were identical to the target, and in Experiment 2 , the percentage was almost as high (35\%). In Experiment 3 , all but one of the nontargets differed from the target on $87.5 \%$ of trials, whereas all but one of the nontargets was identical to the target on only $12.5 \%$ of the trials. This was done in an effort to encourage subjects to focus their attention on the target location; failure to do so would be highly detrimental to their overall performance. It also provided little incentive for subjects to attempt to use multiple identical characters as the basis of their responses, which also would be detrimental to performance. To further maximize the identification of the correct character as the target, one group of subjects received red targets with green nontargets. The other group had white targets and nontargets, like those used in the other experiments.

In addition, subjects gave a confidence rating after each identification response (Bonnel \& Miller, 1994) to allow a determination of whether subjects would display a difference in confidence, or a difference in accuracy associated with particular levels of confidence, for the two MATCH-T conditions.

\section{Method}

The method was similar to that in Experiment 1. Because the MATCH-T effect was smaller in Experiment 2 when there were only four target locations, in Experiment 3, cues and targets could appear 
Table 1

Mean Proportion Correct for Each MATCH-T, and Mean Proportion Correct and Mean Proportion in Error for Each MATCH-NT Within Each MATCH-T in Experiment 2

\begin{tabular}{|c|c|c|c|c|c|c|}
\hline MATCH-T & $\begin{array}{c}p(\text { corr }) \\
\text { for each } \\
\text { MATCH-T }\end{array}$ & MATCH-NT & $\begin{array}{c}p(\text { corr }) \\
\text { for each } \\
\text { MATCH-NT }\end{array}$ & $\begin{array}{c}p \text { (corr) } \\
\text { derived from } \\
\text { Equation } 1 \\
\end{array}$ & $\begin{array}{c}p \text { (responses that } \\
\text { match each } \\
\text { MATCH-NT) }\end{array}$ & $\begin{array}{c}p \text { (matching } \\
\text { error } \\
\text { responses) }\end{array}$ \\
\hline \multirow[t]{8}{*}{0} & .627 & $3,2,2$ & .625 & .63 & & \\
\hline & & 2 & & & .115 & .306 \\
\hline & & 2 & & & .115 & .306 \\
\hline & & 3 & & & .146 & .388 \\
\hline & & $7,0,0$ & .628 & .63 & & \\
\hline & & 0 & & & .100 & .268 \\
\hline & & 0 & & & .100 & .268 \\
\hline & & 7 & & & .173 & .464 \\
\hline \multirow[t]{12}{*}{1} & .637 & $2,2,2$ & .631 & .64 & & \\
\hline & & 2 & & & .123 & .333 \\
\hline & & 2 & & & .123 & .333 \\
\hline & & 2 & & & .123 & .333 \\
\hline & & $3,3,0$ & .646 & .64 & & \\
\hline & & , & & & .093 & .268 \\
\hline & & 3 & & & .127 & .360 \\
\hline & & 3 & & & .127 & .360 \\
\hline & & $6,0,0$ & .635 & .64 & & \\
\hline & & 0 & & & .098 & .280 \\
\hline & & 0 & & & .098 & .280 \\
\hline & & 6 & & & .169 & .464 \\
\hline \multirow[t]{8}{*}{4} & .664 & $1,1,1$ & .665 & .67 & & \\
\hline & & 6,6 & & & .112 & .333 \\
\hline & & 1 & & & .112 & .333 \\
\hline & & 1 & & & .112 & .333 \\
\hline & . & $3,0,0$ & .665 & .67 & & \\
\hline & & 0 & & & .095 & .286 \\
\hline & & 0 & & & .095 & .286 \\
\hline & & 3 & & & .142 & .427 \\
\hline \multirow[t]{4}{*}{7} & .708 & $0,0,0$ & .708 & .70 & & \\
\hline & & 0 & & & .097 & .333 \\
\hline & & 0 & & & .097 & .333 \\
\hline & & 0 & & & .097 & .333 \\
\hline
\end{tabular}

Note-MATCH-T $=$ nontargets identical to targets. $\mathrm{MATCH}-\mathrm{NT}=$ nontargets different from the target but the same as each other. $p$ (corr) $=$ proportion correct. $p$ (matching error responses) $=$ proportion of error responses that match each MATCH-NT within each MATCH-T. MATCH-NT $=0$ refers to a response to a character that was not in the display.

at any one of eight possible locations. There were two groups of 16 subjects each ( 13 men and 19 women). One group viewed light characters against a dark background, as in the first two experiments (white stimuli). For the other group, the background was dark, but the modified plus target always was red whereas the modified plus nontargets were green; this allowed the subject to distinguish the target from the nontargets (red/green stimuli) more easily. For both groups, 448 of the 512 trials for each subject had MATCH-T = 1 and MATCH-NT $=6$, with the location of the MATCH-T randomly determined. The remaining 64 trials had MATCH-T $=6$ and MATCH-NT $=1$, with the location of the MATCH-NT randomly determined. Within each MATCH-T, the target appeared at each location within each ISI an equal number of times. After each trial, subjects first gave an identification response as in the earlier experiments and then were required to give a confidence rating for that response. To indicate high confidence, medium confidence, or low confidence, the subject pressed the " 8 ," "5," or " 2 " key, respectively.

A general linear models procedure was employed to analyze the confidence ratings, because some subjects failed to use some rating levels for one or both conditions. With white stimuli, 2 subjects never assigned the medium-confidence rating for any response in either MATCH-T condition, and two different subjects did not assign the low-confidence rating to any response in the MATCH-T $=6$ condition. For the red/green stimuli, only one observation for 1 of the sub- jects (medium confidence, MATCH-T $=6$ ) was missing. In all other respects, the method was identical to that in Experiment 2.

\section{Results and Discussion}

Overall analyses. In an analysis of all of the data, there were significant increases in accuracy with increasing ISI $\left[F(3,90)=36.49, M S_{\mathrm{e}}=.0077\right]$ and with increasing number of MATCH-Ts $\left[F(1,30)=28.02, M S_{\mathrm{e}}=.0186\right.$, $p<.001]$. (See Figure 3.) The main effect of groups (white or red/green stimuli) was not significant $(p>.20)$, and the only significant interaction was that of groups $\times$ ISI $\left[F(3,90)=3.81, M S_{\mathrm{e}}=.0077, p<.02\right]$. Tukey (HSD) post hoc test indicates that groups differed significantly in accuracy at ISI $=0(p<.05)$, but at no other ISI.

White stimuli. Analysis of the data for the group with white stimuli (means appearing as open symbols in Figure 3) revealed that accuracy increased with increasing ISI $\left[F(3,45)=30.14, M S_{\mathrm{e}}=.0079, p<.001\right]$ and also that accuracy for MATCH-T $=6(M=0.705)$ was significantly greater than that for MATCH-T $=1[M=0.626 ; F(1,15)=$ $\left.9.93, M S_{\mathrm{e}}=.0202, p<.01\right]$. Note that the descriptive equation developed in Experiment 2, $p$ (corr) $=1-[0.3+$ 
Table 2

MATCH-T and MATCH-NT According to $p$ (Responses That Match Each MATCH-NT) and $p$ (Matching Error Responses) in Experiment 2

\begin{tabular}{cccc}
\hline MATCH-T & MATCH-NT & $\begin{array}{c}p \text { (responses that match } \\
\text { each MATCH-NT) }\end{array}$ & $\begin{array}{c}p \text { (matching error } \\
\text { responses) }\end{array}$ \\
\hline 0 & 0 & .0995 & .268 \\
1 & 0 & .0934 & .268 \\
1 & 0 & .0981 & .280 \\
4 & 0 & .0952 & .286 \\
7 & 0 & .0973 & .333 \\
4 & 1 & .1119 & .333 \\
0 & 2 & .1148 & .306 \\
1 & 2 & .1231 & .333 \\
0 & 3 & .1457 & .388 \\
1 & 3 & .1273 & .360 \\
4 & 3 & .1421 & .427 \\
1 & 6 & .1693 & .464 \\
0 & 7 & .1727 & .464 \\
\hline
\end{tabular}

Note-MATCH-T $=$ nontargets identical to targets. MATCH-NT $=$ nontargets different from the target but the same as each other. $p$ (matching error responses) $=$ proportion of error responses that match each $\mathrm{MATCH}-$ NT within each MATCH-T. MATCH-NT $=0$ refers to a response to a character that was not in the display.

0.01(MATCH-NT)], yields a predicted mean of 0.690 for $\mathrm{MATCH}-\mathrm{T}=6$, and 0.640 with MATCH-T $=1$, which are quite close to the obtained means.

Red/green stimuli. Analysis of the data for the group with red/green stimuli (means appearing as filled symbols in Figure 3) revealed an increase in accuracy with increasing ISI $\left[F(3,45)=9.67, M S_{\mathrm{e}}=.0075, p<.001\right]$ and a larger proportion of correct responses with MATCH-T $=6(M=$ $0.778)$ than with MATCH-T $=1[M=0.676 ; F(1,15)=$ $\left.19.33, M S_{\mathrm{e}}=.0170, p<.001\right]$. Equation 1 underpredicts these means.

Distance effects. For MATCH-T $=1$ with white and red/green stimuli (circles in Figure 4), accuracy decreased significantly as distance from the matching nontarget to the target increased [overall means of $0.691,0.630,0.630$, and 0.622 for 1 vs. 2 vs. 3 vs. 4 characters from the target, respectively; $\left.F(3,90)=17.80, M S_{\mathrm{e}}=.0016, p<.001\right]$. The groups $\times$ distance interaction were not significant.

For MATCH-T $=6$, with white and red/green stimuli (square symbols in Figure 4), there was an increase in accuracy with increasing distance of the nonmatching character from the target [overall means of $0.713,0.701,0.757$, and 0.815 for 1 vs. 2 vs. 3 vs. 4 characters from the target, respectively; $\left.F(3,90)=6.87, M S_{\mathrm{e}}=.0124, p<.001\right]$, and there was a significant groups by distance interaction $\left[F(3,90)=3.02, M S_{\mathrm{e}}=.0124, p<.05\right]$.

Thus, accuracy clearly decreased with increases in distance of the MATCH-T from the target with MATCH-T $=1$ and increased with increases in distance of the MATCHNT from the target with MATCH-T $=6$, for both groups. These effects are in contrast to the lack of distance effects in Experiment 1. However, in that experiment, there were only distances available with MATCH-T $=4$ or less. There was a small significant decrease with distance for MATCH-T $=1$. However, the large increase with distance in Experiment 3 was only shown with MATCH-T $=6$, in which there was an increase in accuracy as the distance of the target from the character that does not match the target increased. Thus, this effect provides evidence for more influence of the nonmatching character than of the matching character.

One possible explanation for the distance results in Experiment 3 is that an attentional gradient exists, beginning at the precued location and extending across uncued locations. Another possibility is that subjects occasionally misfocused attention on a single item, and when that occurred, the likelihood of a given location's being incorrectly attended decreased with increasing distance from the target.

Confidence ratings. Confidence ratings for the two groups were analyzed separately on the basis of correct responses for each confidence level within each MATCH-T. Accuracy of identity responses decreased significantly with level of rating for both the white stimuli [high-confidence rating, $M=0.743$; medium-confidence rating, $M=0.546$; and low-confidence rating, $M=0.436 ; F(2,69)=44.30$, $\left.M S_{\mathrm{e}}=.017, p<.001\right]$ and the red/green stimuli [high confidence, $M=0.804$; medium confidence, $M=0.564$; and low confidence, $M=0.522 ; F(2,74)=24.96, M S_{\mathrm{e}}=.030$, $p<.001]$. The interaction between MATCH-T and confidence levels was not significant in either case $(F<1)$.

When the confidence ratings of both groups, white and red/green stimuli, were analyzed together, there was neither a confidence nor a confidence $\times$ MATCH-T interaction with groups $(p>.10)$. Thus, confidence did not vary with MATCH-T group. Even when subjects were confident of their responses, there was still a MATCH-T effect.

Summary. The present results are consistent with those of the immediately preceding experiment, although a strong attempt was made to discourage subjects from reporting an identity that they knew to be that of a nontarget as that of the target. On $87.5 \%$ of the trials, MATCH-T $=1$ and MATCH-NT $=6$; therefore, any identity reported that was known to be that of the nontarget would likely be incorrect. Nevertheless, the MATCH-T effect remained.

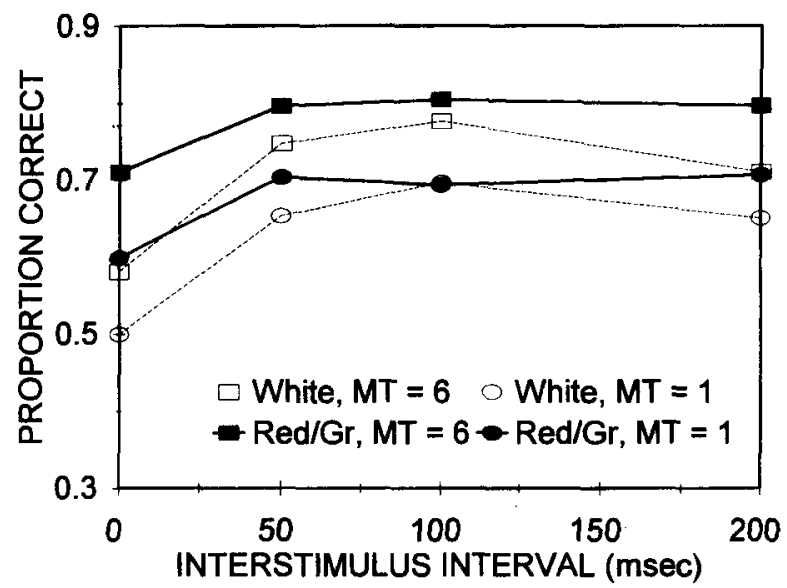

Figure 3. Mean proportion correct for each MATCH-T by cue color and interstimulus interval in Experiment 3. White cues, open symbols and dashed lines; Red/Green cues, closed symbols and solid lines; $\mathrm{MATCH}-\mathrm{T}=6$, squares; $\mathrm{MATCH}-\mathrm{T}=1$, circles. 


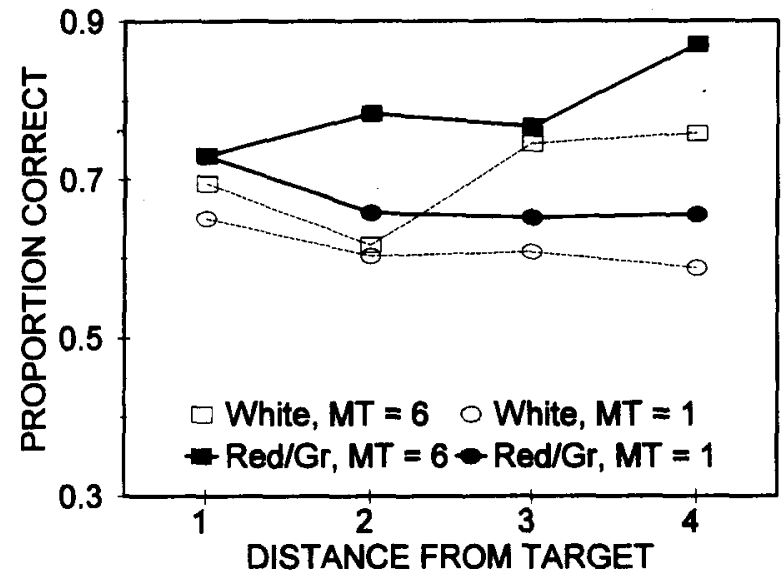

Figure 4. Mean proportion correct as a function of distance of MATCH-T from target for MATCH-T $=1$ and distance of MATCH-NT from target for MATCH-T $=6$ by cue color in Experiment 3. White cues, open symbols and dashed lines; Red/ Green cues, closed symbols and solid lines; MATCH-T $=6$, squares; MATCH-T $=1$, circles.

To one group of subjects, the nontargets were presented in a different color from the targets (green instead of red), to help ensure that a nontarget would not be mistakenly thought to be the target. Although presenting contrasting color stimuli did improve performance, it did not reduce the significant MATCH-T effect.

\section{GENERAL DISCUSSION}

\section{The Effect of Nontargets That Match the Target}

The data from the these experiments show that there is a strong effect of nontargets on accuracy of responding in an identification task under certain conditions. This effect occurs even when the nontargets are separated from the targets by more than $3^{\circ}$ and when they are potentially confusable with the targets (Goolkasian \& Garver, 1993). Thus, multiple identical characters from uncued locations influence responses in a location-cuing paradigm. These results suggest that varying the number of nontargets identical to the target (MATCH-T) between 0 and 1, as was done in the study by Yantis and Johnston (1990), may not allow the MATCH-T effect to emerge. However, with eight characters in the array, which allows for additional MATCH-T conditions, a significant effect of MATCH-T is produced. This MATCH-T effect was found in all three experiments reported here, in our earlier studies, and in new experiments in our laboratory; in these experiments, observers were tested in a single session. It is not known whether the same effect would be found with very practiced observers, but similar effects were found with one experienced observer who received five sessions in the initial task.

With unpracticed subjects, one could not be certain that the cue would be used as directed. Nevertheless, two factors lend credence to a supposition that these observers were shifting their attention to the cued location: (1) There was a significant ISI effect in each experiment, which would be expected if attention were being refocused on the cued location. (2) The removal of trials in which the observers did not know the cued location (Experiment 1) did not change the results. Of course, correctly identifying the location does not mean that the observer always used the cue correctly. However, a new experiment was conducted in our laboratory, in which MATCH-T was either 1 or 6 (with 6 or 1 nontargets, respectively, that did not match the target), or a condition in which there was one nontarget that matched the target and six plus signs (MATCH-T = $1+$ ). There was again higher accuracy with MATCH-T $=6$ than when MATCH-T $=1$ ( $15 \%$ difference; mean proportion correct for 16 subjects $=.636$ and .542 , respectively). The important new data, however, were that subjects reported nearly as many targets correctly when six nontargets were plus signs as when six nontargets matched the target (mean proportion correct $=.609$ ). The results of this experiment are not consistent with the assumption that accuracy differences in the different MATCH-T conditions are due to the fact that subjects occasionally misattend the target location and instead attend a nontarget location and report the nontarget's identity as the target identity. Accuracy in the MATCH-T $=1+$ condition was closer to the scores in the MATCH-T $=6$ condition, rather than only slightly higher than in the MATCH-T $=1$ condition, as would be predicted from such an assumption. Performance seems to be affected instead by the degree to which identities in the display as a whole are consistent or inconsistent with that of the target.

These results provide another example of how stimulusdriven processes (or bottom-up effects) can interact with goal-driven processes (top-down effects) in the allocation of attention. Wolfe, Cave, and Franzel (1989) proposed that search for a visual stimulus is guided to the most likely target by a combination of bottom-up and top-down processes. In a somewhat different paradigm, Todd and Kramer (1994) reported that a unique irrelevant colored or more luminous target was found more quickly than a nonunique target of the same color or luminance. They suggested that a unique color or luminance could misguide attention; that is, the attentional set (or goal-driven process) was modulated by the uniqueness of the target (a stimulus-driven process).

\section{The Effect of Nontargets That Do Not Match the Target}

In Experiment 2, in which the effect of the nontargets that differed from the target (MATCH-NT) was analyzed, there was a significant effect of MATCH-NT, but it was independent of MATCH-T. Within any one MATCH-T, there were no differences in MATCH-NT. Thus, any effect of MATCH-NT on correct trials is accounted for by the MATCH-T effect. However, there were systematic differences on incorrect trials in the proportion of total errors in which the response was the same as that for a group of matching nontargets (for instance, MATCH-NT $=6$ or 7 had a larger proportion of total errors than did MATCH$\mathrm{NT}=1$, Table 2, column 4). Thus, nontargets do affect responses even when, as in the current experiments, the cue is $100 \%$ valid--that is, when it always indicates the correct target location. 
If Eriksen et al. (1990) are correct in proposing that there are two independent modes of attention (automatic and focused), it is possible that automatic processing of nontargets occurs. If nontargets do not match the target, the focal process may overrule the result of any automatic processing, which would account for the null effect of MATCH-NT within MATCH-T on correct responses. On the other hand, if the nontargets match the targets, the automatic process would add support to the focal process in identifying the target.

The fact that there was a MATCH-NT effect on the incorrect responses would suggest that on some small proportion of those trials, observers did not focus attention on the cued location. Quite possibly, on some trials attention was not successfully focused on any character, and this occurrence may be reflected in the baseline guessing rate of approximately. 10 (see Equation 1) that was observed when the response was to a character not in the display (MATCH$\mathrm{NT}=0$; Table 2, column 3 ). On some other trials, the automatic processing of nontargets that matched each other may have supported an incorrect response, and this may have become part of the .01 increase with MATCH-NT in proportion of responses that match the nontarget (Equation 1; Table 2, column 3).

Alternatively, attention may have been focused on a character that was not the target, so that the response was based on an attended nontarget, rather than an automatically processed nontarget. If the nontarget matched the target, the response was correct, or if the nontarget was different from the target, the response was incorrect. The extent to which responses resulted from automatic processing versus misfocusing of attention on a nontarget cannot be determined precisely with the present data, although a strong incentive for the focus of attention was provided in Experiment 3.

\section{Metaphors for Attention}

These data are also relevant to the concepts of how attention is spatially allocated to a visual field. Of the three metaphors for allocation of attention discussed in the introduction, only the gradient filter is consistent with all of the data presented here. Obviously a spotlight metaphor cannot be accurate, because the nontargets had an effect on accuracy of identification in the three experiments reported here as well as in additional unreported experiments. If attention had been allocated in a unitary fashion that started at fixation and then moved across the display directly to the cued location, nontargets outside of that path would have had no effect.

If attention had been allocated like a zoom lens, there should have been an interaction between MATCH-T effects and ISI, because nontargets should only have been influential before attention had time to build at the target location. However, there was no significant MATCH-T $\times$ ISI interaction in the three experiments (see Figures 1-3). Thus, the MATCH-T effect did not lessen at longer ISIs as would be the case if attention first was broadly allocated and then narrowed with longer delays as the zoom lens metaphor would predict. The zoom lens metaphor received little, if any, support in any of the experiments.

On the other hand, the gradient-filter metaphor for attention (Cheal et al., 1994) is consistent with all of the data. According to this metaphor, attention changes the "permeability" of multiple filters. The permeability is reflected in the quantity of information that can flow from the visual display to the percept. The distribution and amount of permeability can be a function of the task and can vary across the field. In the location-cuing paradigm, it is thought that most of the attention is focused on the cued location, but that if a total capacity has not been reached, some attention may be allocated to nontarget areas. This is consistent with the data collected here. The increase in slope of accuracy as a function of ISI suggests that the majority of attention continued to focus on the cued location until asymptote at $100-200 \mathrm{msec}$. However, the characters at nontarget locations also affected responses, which suggests that some attention was allocated to these other locations.

The gradient-filter metaphor suggests that attention can be shared across locations within a trial; that is, it can be simultaneously allocated to more than one location. An alternative explanation for the present data is that on some trials attention was focused on a noncued location, thus resulting in intrusions from nontargets. This can be called switching of attention over trials. Our comments above that argue against attention's sometimes focusing on a noncued character can also be taken as arguments against switching of attention.

In order to investigate the issue of switching versus sharing, it may be necessary to have cues that indicate the wrong location on some proportion of the trials. In the present research, all trials had valid cues. But in Gottlob, Cheal, and Lyon (1995; see also Gottlob, 1995), the proportion of valid cues was varied. This variation allowed the application of a formal model of sharing attention that fit locationcuing data better for most observers than did a similar model of switching attention across trials. Unfortunately, in the Gottlob et al. studies, the number of matching and nonmatching nontargets was not manipulated.

The gradient-filter metaphor does not predict whether there will be an interaction between MATCH-T condition and ISI, because the interaction would depend on the task demands. However, in the other experiments with varying numbers of matching nontargets, resources may accumulate across locations of matching nontargets.

\section{Conclusions}

The present data clearly demonstrate that an irrelevant item or items presented outside the focus of attention can affect performance on an attended target. Our analyses suggest the following: (1) an irregular spread of attention (consistent with the gradient-filter metaphor) so that small amounts of attention are at uncued locations with the most attention at the cued location, and (2) an automatic process that may retrieve information from nontarget locations, which then influences the percept. 


\section{REFERENCES}

BONNEL, A.-M., \& MiLler, J. (1994). Attentional effects on concurrent psychophysical discriminations: Investigations of a sample-size model. Perception \& Psychophysics, 55, 162-179.

ChEAL, M., \& LYON, D. (1989). Attention effects on form discrimination at different eccentricities. Quarterly Journal of Experimental Psychology, 41A, 719-746.

ChEAL, M., \& LyON, D. R. (1992). Attention in visual search; Multiple search classes. Perception \& Psychophysics, 52, 113-138.

Cheal, M., Lyon, D. R., \& Gottlob, L. R. (1994). A framework for understanding the allocation of attention in location-precued discrimination. Quarterly Journal of Experimental Psychology, 47A, 699-739.

ERIKSEN, C. W., \& ST. JAMES, J. D. (1986). Visual attention within and around the field of focal attention: A zoom lens model. Perception \& Psychophysics, 40, 225-240.

EriKSEN, C. W., WebB, J. M., \& Fournier, L. R. (1990). How much processing do nonattended stimuli receive? Apparently very little, but ... Perception \& Psychophysics, 47, 477-488.

GoolKasian, P., \& GaRVER, D. K. (1993). Evidence for processing letters at uncued locations. American Journal of Psychology, 108, 235-253.

GoTrLOB, L. R. (1995). Modeling sharing versus switching in visual attention. Unpublished doctoral dissertation, Arizona State University.

GotTlob, L. R., Cheal, M., \& Lyon, D. R. (1995). Cue validity manipulation in a location-cuing task: Sharing versus switching models for attention. Manuscript submitted for publication.

Johnston, W. A., \& DARK, V. J. (1986). Selective attention. Annual Review of Psychology, 37, 43-75.

LABERGE, D., \& BROWN, V. (1986). Variations in size of the visual field in which targets are presented: An attentional range effect. Perception \& Psychophysics, 40, 188-200.

LABERGE, D., \& BRown, V. (1989). Theory of attentional operations in shape identification. Psychological Review, 96, 101-124.

MilleR, J. (1987). Priming is not necessary for selective-attention failures: Semantic effects of unattended, unprimed letters. Perception \& Psychophysics, 41, 419-434.

Paquet, L., \& Lortie, C. (1990). Evidence for early selection: Precuing target location reduces interference from same-category distractors. Perception \& Psychophysics, 48, 382-388.

Pashler, H., \& Badgio, P. C. (1985). Visual attention and stimulus identification. Journal of Experimental Psychology: Human Perception \& Performance, 11, 105-121.

PosNer, M. I. (1980). Orienting of attention. Quarterly Journal of Experimental Psychology, 32, 3-25.

TODD, S., \& KRAMER, A. F. (1994). Attentional misguidance in visual search. Perception \& Psychophysics, 56, 198-210.

Wolfe, J. M., CaVe, K. R., \& Franzel, S. L. (1989). Guided search: An alternative to the feature integration model for visual search. Journal of Experimental Psychology: Human Perception \& Performance, 15, 419-433.

Wright, R. D., Katz, A. N., \& Hughes, E. A. (1993). Inattention and the perception of visual features. Acta Psychologica, 83, 225-235.

YANTIS, S., \& JoHNSTON, J. C. (1990). On the locus of visual selection: Evidence from focused attention tasks. Journal of Experimental Psychology: Human Perception \& Performance, 16, 135-149.

(Manuscript received April 28, 1994; revision accepted for publication July 26,1995 .) 